\title{
Reliability and validity of the Persian version of 5-D itching scale among patients with chronic kidney disease
}

\author{
Amin Kordi Yoosefinejad ${ }^{1}$, Fatemeh Karjalian², Marzieh Momennasab ${ }^{3^{*}}$ (D) and Shahrokh Ezzatzadegan Jahromi ${ }^{4}$
}

\begin{abstract}
Background: Hemodialysis is considered a major therapeutic method for patients with chronic kidney disease. Pruritus is a common complaint of hemodialysis patients. The 5-D pruritus scale is amongst the most common tools to evaluate several dimensions of itch. Psychometric properties of the 5-D scale have not been evaluated in Persian speaking population with hemodialysis; hence, the objective of this study was to assess reliability and validity of the Persian version of the scale.

Methods: Ninety hemodialysis patients (men: 50, women: 40, mean age: 54.4 years) participated in this crosssectional study. The final Persian version of 5-D scale was given to the participants.

Tests Compared: One-third of the participants completed the scale twice within 3-7 days apart to evaluate testretest reliability. Other psychometric properties including internal consistency, absolute reliability, convergent, discriminative and construct validity, floor/ceiling effects were also evaluated.

Results: The Persian 5-D scale has strong test-retest reliability $(I C C=0.98)$ and internal consistency (Cronbach's alpha $=0.99$ ). Standard error of measurement and minimal detectable change were 0.33 and 0.91 , respectively. Regarding convergent validity, the scale had moderate correlation with numeric rating scale $(r=0.67)$ and quality of life questionnaire related to itch $(r=0.59)$. Exploratory factor analysis revealed two factors within the scale. No floor or ceiling effect was found for the scale.

Conclusion: The Persian version of 5-D the itching scale is a brief instrument with acceptable reliability and validity. Therefore, the scale could be used by experts, nurses, and other health service providers to evaluate pruritus among Persian speaking hemodialysis patients.
\end{abstract}

Keywords: 5-D pruritus scale, Validity, Reliability, Hemodialysis, Chronic kidney disease

\section{Background}

Chronic kidney disease (CKD) is a public health problem across the word. Hemodialysis is a major therapeutic method for many patients with CKD especially for those at end-stage renal disease with a prevalence of 22 to $84 \%$ [1]. Pruritus is a common complication among

\footnotetext{
* Correspondence: momennasab@sums.ac.ir

${ }^{3}$ Department of Nursing, School of Nursing and Midwifery, Shiraz University of Medical Sciences, Zand St., Namazee Sa, Shiraz 7193613119, Iran Full list of author information is available at the end of the article
}

hemodialysis patients [2]. Pruritus is an unpleasant sensation, accompanied by desire to scratch the affected area [1] affecting up to $46 \%$ of hemodialysis patients [3]. The prevalence of pruritus among Iranian hemodialysis patients was reported as 41.9 to $48.3 \%$ [4]. Not only does the pruritus impact quality of life in hemodialysis patients, but also it has high psychological burdens [5]. Several scales have been developed to quantify the rate of pruritus. The Visual Analogue Scale (VAS), the Eppendorf Itch Questionnaire [6], the Skindex [7], the 
Itch medical outcome study [8], and the 5-D pruritus scale are among the most popular tools used to evaluate the rate of itching. Currently, there is no consensus on a universal scale which would be able to evaluate severity, degree, duration, and impact on quality of life among hemodialysis patients.

The 5-D itch scale was first developed by Elman et al. as a new measure of itching [9]. It was validated with a numerical rating scale (NRS) in patients with human immunodeficiency virus, skin, liver or kidney disease [9]. In contrast to unidirectional scales such as NRS and VAS, the 5-D pruritus scale is a multidimensional measure. Previously 5-D itch scale was used as an assessment tool to evaluate multidimensional aspects of pruritus among hemodialysis patients [10]. Considering that pruritus as one of the most common and frustrating symptoms among hemodialysis patients, evaluating different dimensions of itching seems mandatory. Moreover, there is lack of an agreed-upon valid multidimensional scale for Persian-speaking experts and health care providers to evaluate pruritus among hemodialysis patients. Therefore, the objective of this study was to evaluate the validity and reliability of the Persian version of 5-D pruritus scale.

\section{Methods}

This cross-sectional study was part of a more extensive study conducted between August 2018 and February 2019 at dialysis centers affiliated with Shiraz University of Medical Sciences, Shiraz, Iran. The study was approved by the Ethics Committee of the Vice Chancellery of Shiraz University of Medical Sciences in concordance with the standards of Helsinki declaration (Ethics number: IR.SUMS.REC.1396.2.9). Ninety patients were recruited from dialysis centers affiliated with Shiraz University of Medical Sciences, Shiraz, Iran. Patients aged at least 18 years old and received hemodialysis for more than 3 months were eligible to participate in the study. Patients with autoimmune diseases such as systemic lupus erythematous, and those with liver complications were excluded. The sample size was calculated as a minimum of 50 patients based on the ratio between the number of items in scale to participants which is considered as a ratio of 1:10 [11]. All patients signed written informed consent form before participating in the study.

Five dimensions of itching beginning with letter " $D$ " are included in 5-D pruritus scale and are evaluated during the previous 2 weeks. These dimensions include duration; degree; direction; disability; and distribution of itching. The first three items (duration, degree, direction) are single-item domains and are scored from 1 ('less involvement according to the item') to 5 ('most involvement according to the item'). Disability represents a multiple-item domain and includes the effects of itching on daily activities such as sleep, leisure/social activities, housework/errands and work/school. The score for the disability domain is obtained by obtaining the highest score within four evaluated sub domains. The fifth item (Distribution) evaluates the presence of itching within 16 body parts over the previous 2 weeks. Regarding the number of affected parts, five scoring bins are constructed. The sum of $0-2$ is considered as the score of bin 1 , sum of $3-5=$ score of 2 , sum of $6-10=$ score of 3 , sum of $11-13=$ score of 4 and sum of $14-16=$ score of 5. 5-D scores range from 5 (no pruritus) to 25 (most severe pruritus).

\section{Translation process}

To translate the 5-D itching scale for Persianspeaking dialysis patients, we followed the guidelines served as a template for the translation and crosscultural adaptation in medical literature introduced by Beaton et al. [12].

Initial translation: The English version of the 5-D scale was translated into Persian by two native bilingual translators independently. One translator was an experienced nurse who was aware of the concept of the scale.

Table 1 Demographic data of the participants $(n=90)$

\begin{tabular}{ll}
\hline Demographics & $\mathbf{n}(\%)$ \\
\hline Sex & \\
Men & $50(55.6)$ \\
Women & $40(44.4)$ \\
Age (years) & \\
Range & 39 \\
mean & 54.40 \\
Median & 58 \\
Education & \\
Illiterate & $50(55.6)$ \\
primary level (6 years of education) & $6(6.7)$ \\
secondary level (12 years of education) & $33(36.7)$ \\
tertiary level ( $\geq$ 16 years of education) & $1(1.1)$ \\
Marital status & \\
Married & $75(83.3)$ \\
Single & $7(7.8)$ \\
Widowed & $4(4.4)$ \\
Divorced & $4(4.4)$ \\
Settlement & \\
Urban & \\
Suburb & $24(26.7)$ \\
Expenses for treatment borne by & $66(73.3)$ \\
Fovernment & $42(46.7)$ \\
\hline & $48(53.4)$ \\
\hline
\end{tabular}


Table 2 Scoring as per the domains mentioned in the Persian version of 5-D itching scale

\begin{tabular}{l} 
Variable \\
\hline Duration (Mean score $=3.29 \pm 0.98$ ) \\
Less than $6 \mathrm{~h}$ a day \\
$6-12 \mathrm{~h}$ a day \\
$12-18 \mathrm{~h}$ a day \\
$18-23 \mathrm{~h}$ a day \\
All day
\end{tabular}

Degree (Mean score $=4.18 \pm 0.59)$

Not present

Mild

Moderate

Severe

Unbearable

Direction (Mean score $=3.63 \pm 0.66$ )

Completely resolved

Much better, but itching is still present

Little bit better but still presents

Unchanged

Getting worse

N (\%)

$3(3.3)$

$12(13.3)$

$44(48.9)$

$18(20)$

$13(14.4)$

$0(0)$

$0(0)$

$9(10)$

$55(61.1)$

$26(28.9)$

$8(8.9)$

$42(46.7)$

$39(43.3)$

$1(1.1)$

$0(0)$

Disability (Mean score $=9.05 \pm 2.79$ ) $[$ Minimum $=4$, Maximum $=16$ ]

Sleep (Mean score $=3.51 \pm 1.25$ )

Never affects sleep

$8(8.9)$

Occasional delays falling asleep

$13(14.4)$

Frequent delays falling asleep

$16(17.8)$

Delays falling asleep and occasionally wakes me up at night 31 (34.4)

Delays falling asleep and frequently wakes me up at night

Leisure/social activity (Mean score $=2.11 \pm 0.77$ )

Never affects leisure/social activity

$20(22.2)$

Rarely affects leisure/social

Occasionally affects leisure/social

Frequently affects leisure/social

Always affects leisure/social

Housework/errands (Mean score $=1.72 \pm 0.72$ )

Never affects house work/errands

Rarely affects house work/errands

Occasionally affects house work/errands

Frequently affects house work/errands

Always affects house work/errands

Work/School (Mean score $=1.71 \pm 0.76$ )

Not applicable

Never affects Work/School

Rarely affectsWork/School

Occasionally affectsWork/School

Frequently affectsWork/School

$40(44.4)$

$11(12.2)$

$1(1.1)$

$0(0)$

$40(44.4)$

$1(1.1)$

$36(40)$

$11(12.2)$

$2(2.2)$
Table 2 Scoring as per the domains mentioned in the Persian version of 5-D itching scale (Continued)

\begin{tabular}{ll}
\hline Variable & $\mathbf{N}(\%)$ \\
\hline Always affectsWork/School & $0(0)$ \\
Distribution (Mean score= $3.00 \pm 0.75)$ [Minimum=1, Maximum $=5]$ \\
Scoring Bin 1 & $1(1.1)$ \\
Scoring Bin 2 & $19(21.1)$ \\
Scoring Bin 3 & $52(57.8)$ \\
Scoring Bin 4 & $15(16.7)$ \\
Scoring Bin 5 & $3(3.3)$ \\
Total score (Mean score $=17.69 \pm 3.03)$ & \\
$6-10$ & $0(0)$ \\
$11-19$ & $62(68.9)$ \\
$20-25$ & $28(31.1)$ \\
\hline
\end{tabular}

Another was neither medical staff nor informed of the concepts of the scale.

Synthesis of the translations: Translators and a recording observer synthesized the initial results. Comparing the original scale with translations, a written report documenting the synthesis process was provided. Hence, a finalized forward translation of 5-D scale was provided.

Back translation: forward translation of the 5-D scale was given to two bilingual translators whose native language was English and were not medical staff. The translators were not aware of the concepts of scale.

Expert committee: The aim of this stage was to consolidate all the versions and to develop pre-final version of scale. Decisions were made on semantic, idiomatic, experiential, and conceptual equivalences. The committee comprised of the translators, two expert nurses, a health professional and a methodologist.

Test of the pre-final version: Eight hemodialysis patients with itching completed the scale and had a cognitive interview about the concept of items and the provided responses. 1 Items were easily comprehended by interviewees.

Submission of documentation to the coordinating committee for appraisal of the adaptation process: Expert committee came to a consensus on the final adapted version of scale.

\section{Reliability}

Relative reliability of the 5-D scale was evaluated with the test-retest intraclass correlation coefficient (ICC) and internal consistency of the scale. Thirty patients completed the 5-D scale twice, 3 to 7 days apart. Internal consistency was evaluated with Cronbach's alpha.

Absolute reliability of the scale was evaluated with calculating standard error of measurement (SEM) and minimal detectable change (MDC).

SEM is calculated as follows: 


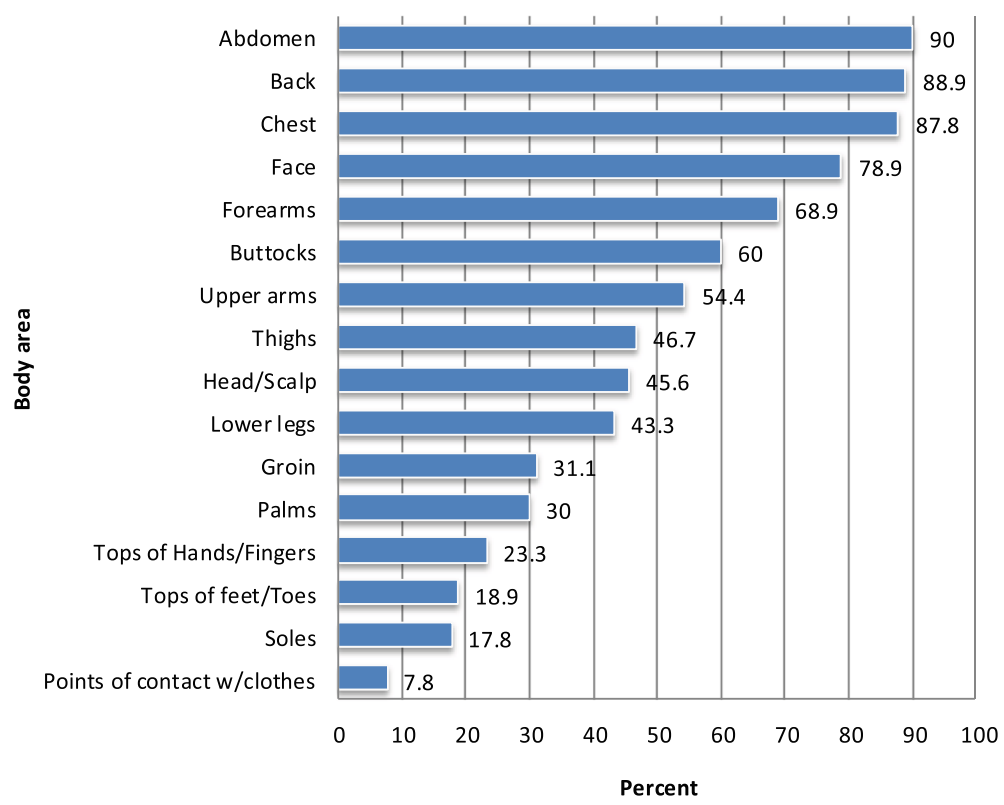

Fig. 1 Distribution of itch percentage among different regions of body in patients receiving hemodialysis

$$
\mathrm{SEM}=\mathrm{SD} \times \sqrt{ } 1-\mathrm{ICC}
$$

"SD" stands for standard deviation and ICC stands for intraclass correlation coefficient. MDC is calculated employing the formula: $\mathrm{MDC}=1.96 \times \sqrt{ } 2 \times \mathrm{SEM}$ [13].

\section{Validity}

Convergent validity of the 5-D scale was evaluated with a numeric rating scale (NRS) and scales. The NRS is a uni-dimensional scale comprised of 11 points. Achieving zero points is interpreted as "no pruritus",1-3 points on NRS is interpreted as mild itch, 4-6 points as moderate itch, 7-8 points as severe and 9 points or more points is interpreted as severe pruritus [14]. NRS $\geq 4$ was considered as symptomatic pruritus [14].

The Itchy QoL questionnaire is a standard, common self-reported instrument designed to evaluate quality of life among patients with pruritus. It was first developed by Desai et al. (2008) and consists of 26 questions evaluating three different major aspects of the quality of life in patients with pruritus [15]. The first aspect is about symptoms experienced by the individual and includes the first six questions. Questions 7 to 16 evaluate "functioning" dimension and questions 17 to 26 evaluate the "emotion" dimension of the individuals. Each question is designed as a Likert type item from 1 (never) to 5 (always). Scores are considered separately within each dimension and also totally as the aggregate score of itchy QoL questionnaire. Score range is 26 to 130 ; the higher the score the greater the quality of life. Psychometric properties of the Persian version of Itchy QoL were evaluated by Tari et al. [16]. Construct validity of the scale was evaluated with exploratory factor analysis using principal component analysis and varimax rotation [17]. The Eigenvalues greater than one were considered as having a significant contribution in explaining the

Table 3 Reliability measures of the Persian version of 5-D scale

\begin{tabular}{llllll}
\hline Domains & Test Median & Retest Median & Corrected item total correlation & Cronbach's alpha if item deleted & ICC \\
\hline Duration & 3 & 3 & 0.62 & 0.85 & 0.98 \\
Degree & 2 & 2 & 0.60 & 0.85 & 0.99 \\
Direction & 3 & 3 & 0.47 & & \\
Disability & & & 0.86 & 0.99 \\
Sleep & 2 & 2 & 0.70 & 0.85 & 0.97 \\
Leisure/Social life & 4 & 4 & 0.68 & 0.85 & 0.98 \\
House work & 4 & 4 & 0.65 & 0.85 & 0.99 \\
$\quad$ Work/School & 4 & 4 & 0.61 & 0.86 & 0.99 \\
Distribution & 2 & 3 & 0.48 & &
\end{tabular}


overall model variation. Sampling adequacy was examined using Bartlett's test of sphericity and KaiserMayer-Olkin (KMO). A KMO value greater than 0.6 was considered acceptable, and 0.80 indicates a good level of compatibility of the variables within the test.

To determine discriminative validity, the MannWhitney $U$ test was used to evaluate the scores of the participants at baseline and re-test to observe no significant difference between the results [18].

Floor and ceiling effects were calculated for the Persian version of 5-D scale. These are defined as if more than $15 \%$ of the participants achieve either the least (floor) or the greatest (ceiling) scores.

\section{Results}

A total of 90 patients undergoing hemodialysis was recruited. Demographic characteristics of the participants are summarized in Table 1 . The mean score of the Persian version of the 5 -D scale was $17.69 \pm 3.03$ with scores ranging from 11 to 25 . The mean time for receiving hemodialysis was $7.04 \pm 3.01$ years (Range: 13 years) and the mean itching duration was $21.39 \pm 9.20$ months (range: 41 months). Scoring as per the domains mentioned in the Persian version of the 5-D sitching scale is shown in Table 2. Regarding the NRS grading system for itch, $11(12.2 \%)$ of the participants had moderate degree of itching, $50(55.6 \%)$ of the individuals had severe itching and $29(32.2 \%)$ of the participants had very severe itching. It means all the participants had symptomatic pruritus (NRS $\geq 4)$. Considering the distribution of itch, the abdomen had the most considerable percentage of itch (90\%) while contact with clothes had the least percentage (7.8\%). Distribution percentage of various parts of body is exhibited in Fig. 1.

\section{Reliability}

ICC between the test and retest of the Persian version of the 5-D scale was 0.98 (95\% confidence interval: 0.960.99) and the overall Cronbach's alpha of the scale was 0.99 . The SEM and MDC of the Persian version of the scale were calculated as 0.33 and 0.91 respectively. Further reliability measures are shown in Table 3.

\section{Validity}

The Pearson correlation coefficient between the Persian version of the 5-D scale and the NRS was moderate $(r=0.67, p<$ 0.001). Also, the Persian 5-D had a moderate degree of correlation with Itchy-QoL questionnaire $(r=0.59, p<0.001)$.

Exploratory factor analysis revealed the Persian version of the 5-D scale had two factors. One factor included duration, degree, direction, and sleep items while the other factor comprised of social, housework, school/ work and distribution items. These factors explained $65.22 \%$ of the total observed variance. $[\mathrm{KMO}=0.78$,
Bartlett's test of sphericity was significant. (Chi-square= $371.60, d f=28, p<0,001)]$. The scree plot of the exploratory factor analysis is depicted in Fig. 2. The scree plot is a commonly used graphical method for determining the number of the components to extract.

As can be observed from Fig. 2, two factors ( $\mathrm{x}$-axis) could be extracted from the Persian version of the 5-D itching scale with Eigenvalues greater than one (y-axis) [19].

Discriminative validity revealed no significant difference between the achieved scores of the dimensions of the 5 -D itching score at baseline $(n=90)$ and re-test $(n=30)$. The comparison of the dimensions between test and re-test is summarized in Table 4.

Considering the least $(11,2.2 \%)$ and the highest (25, $1.1 \%)$ achieved scores of the Persian version of the 5-D scale, neither a floor nor ceiling effect was observed in the scale.

\section{Discussion}

The 5-D itching scale is a commonly used patient reported outcome measure in pruritus which was validated into six different languages. The aim of this study was to assess the reliability and validity of the Persian version of the 5-D itching scale among Persian-speaking patients receiving hemodialysis.

The Persian version of the 5-D itch scale revealed excellent internal consistency (Cronbach's alpha $=0.99$ ) and excellent agreement for test-retest reliability (ICC= 0.98). Compared with the Indonesian [20] (Cronbach's alpha $=0.67)$, Malaysian (Cronbach's alpha=0.86) [21], Urdu version [1] (Cronbach's alpha $=0.91$ ), and Arabic version [22] (Cronbach's alpha $=0.85$ ) of the $5-\mathrm{D}$ itch scale, the Persian version showed a higher internal consistency. The original version of the scale reported the internal consistency as 0.73 [9]. The Japanese version of the scale did not report the internal consistency [23]. In line with Urdu ( $\mathrm{ICC}=0.91)$, Arabic $(\mathrm{ICC}=0.85)$, and the original version ( $\mathrm{ICC}=0.96$ ), the Persian version of the 5-D scale showed excellent test-retest reliability. We also evaluated the absolute reliability of the Persian version with calculating SEM and MDC. To our best knowledge, no other version of the scale has evaluated the absolute reliability; so, our findings could not be compared to other versions of the scale.

The Persian version of the 5 -D scale had a moderate degree of correlation with both NRS $(r=0.67)$ and the Persian version of Itchy QoL questionnaires $(r=0.59)$. The original version of the scale also showed a moderate correlation $(r=0.69)$ with the VAS. The Indonesian version had a strong correlation with the dermatology life quality index and the VAS [20]. The Japanese version of the scale showed strong correlation with vas $(r=0.70)$, daytime itchiness $(r=0.64)$, and nighttime itchiness $(r=0.63)$ [23]. Other versions of the scale did 


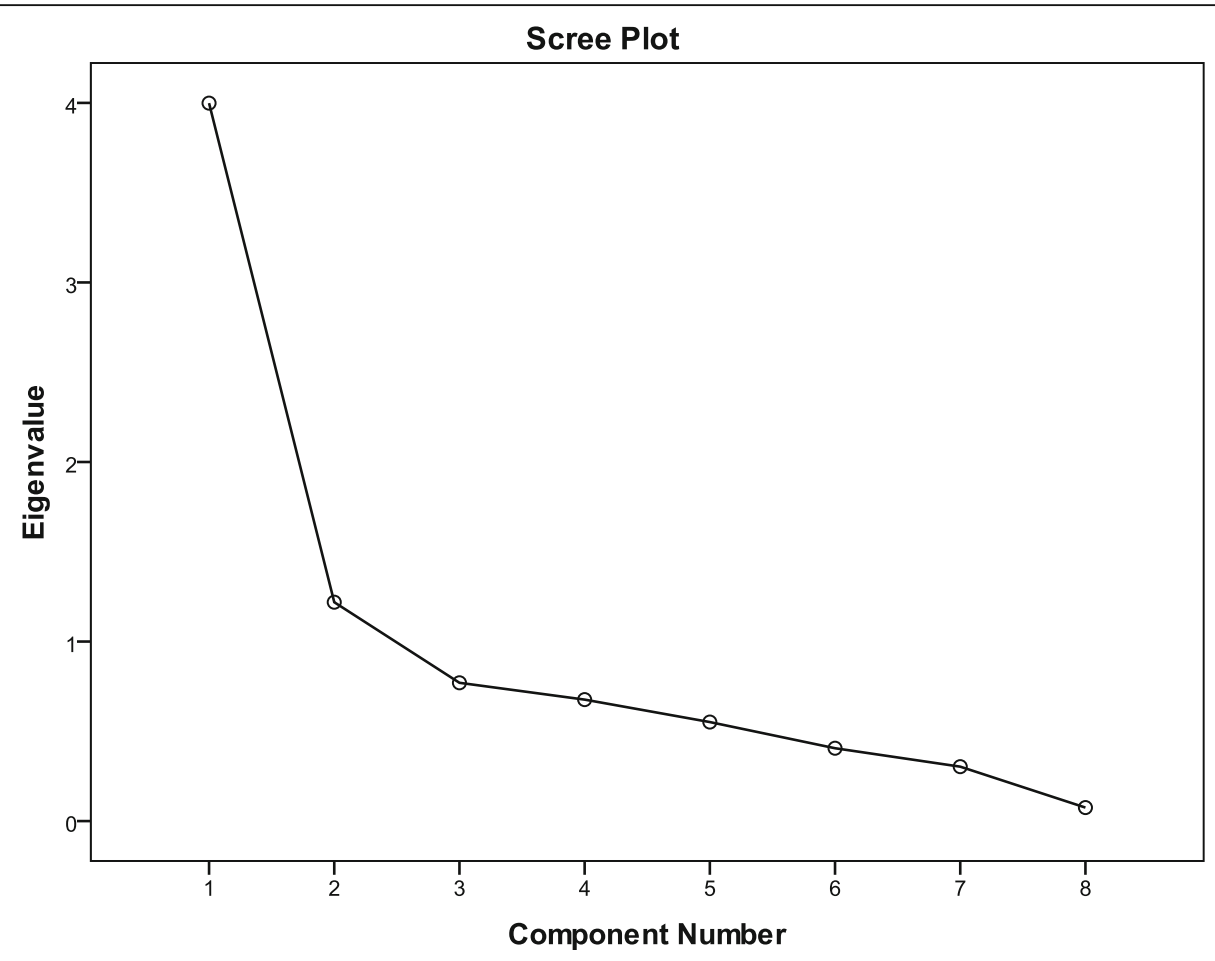

Fig. 2 Scree plot of exploratory factor analysis of the Persian version of the 5-D scale

not evaluate the correlation of the scale with other related instruments. Among the pruritus scales, the Itchy QoL was the only scale to be validated into Persian; hence, we evaluated the convergent validity of the Persian version of the 5-D scale with the abovementioned scales. No other version evaluated the correlation of the 5-D scale with the Itchy QoL.

Exploratory factor analysis revealed two factors for the Persian version of scale. The first factor was "daily routine activities" and the other factor was "pattern of itching". This was in line with the Malaysian version of the scale [21]. The Malaysian version also revealed two factors with similar components for each factor. Moreover, the Urdu version of the scale revealed two factors; however, the loading components were different from those obtained with the Persian version of scale [1]. Although the Arabic version of the scale claimed to have evaluated the factor analysis of the scale, only the values of KMO and Bartlett's test of sphericity were reported and no factor rotation was carried out [22]. The factor analysis was not performed on the other versions of scale. Discriminative

Table 4 Comparison of the scores of the dimensions of the Persian version of 5-D itching scale

\begin{tabular}{|c|c|c|c|c|c|}
\hline \multirow[t]{2}{*}{ Domains } & \multicolumn{2}{|c|}{ Test $(n=90)$} & \multicolumn{2}{|c|}{ Re-test $(n=30)$} & \multirow{2}{*}{$\begin{array}{l}\text { Mann- } \\
\text { Whitney } \\
\mathrm{U} \\
p \text {-value }\end{array}$} \\
\hline & median & IQR (25-75\%) & median & $\mathrm{IQR}^{*}(25-75 \%)$ & \\
\hline Duration & 3 & $1-4$ & 3 & $1-4$ & $>0.99$ \\
\hline Degree & 2 & $1-2$ & 2 & $1-2$ & $>0.99$ \\
\hline Direction & 3 & $2-3$ & 3 & $2-3$ & $>0.99$ \\
\hline \multicolumn{6}{|l|}{ Disability } \\
\hline Sleep & 2 & $1-4$ & 2 & $1-4$ & 0.32 \\
\hline Leisure/Social life & 4 & $3-5$ & 4 & $3-5$ & $>0.99$ \\
\hline House work & 4 & $4-5$ & 4 & $4-5$ & $>0.99$ \\
\hline Work/School & 4 & $4-5$ & 4 & $4-5$ & $>0.99$ \\
\hline Distribution & 2 & $2-3$ & 3 & $2-3$ & 0.06 \\
\hline
\end{tabular}

*: Inter quartile range 
validity of the scale revealed stability of the scale as the participants interpreted items similarly at baseline and retest. To the best of our knowledge, only the Urdu version of the scale evaluated discriminative validity and their results are congruent with ours [1]. The Persian version of the scale revealed either no floor or ceiling effects; however, we could not compare our findings due to dearth of evidence.

To our best knowledge, our study was the first to evaluate absolute reliability and floor/ceiling effects of the 5-D itching scale. Our study had some limitations. We did not evaluate the responsiveness of the scale; thus, future studies are warranted to assess the responsiveness of the scale following several invasive or noninvasive therapies. Also, because of the lack of adequate number of Persian-validated pruritus scales, we performed convergent validity with just the Itchy QoL. Considering our study, future studies have more available instruments to evaluate validity of their tools.

\section{Conclusion}

The Persian version of the 5-D itching scale is a brief tool with acceptable reliability and validity. Therefore, experts, nurses, and other health service providers can use it to evaluate pruritus among Persian speaking patients with CKD.

\section{Supplementary Information}

The online version contains supplementary material available at https://doi. org/10.1186/s12882-020-02220-x.

Additional file 1.

\section{Abbreviations}

CKD: Chronic kidney disease; VAS: Visual analogue scale; NRS: Numerical rating scale; ICC: Intraclass correlation coefficient; SEM: Standard error of measurement; MDC: Minimal detectable change; SD: Standard deviation; QoL: Quality of life; KMO: Kaiser-Mayer-Olkin

\section{Acknowledgements}

This manuscript was extracted from Fatemeh Karjalian M.Sc. thesis on critical care nursing (No:13620). We appreciated the Vice Chancellor for Research Affairs of Shiraz University of Medical Sciences for their financial support and all the patients who participated in our study. The authors are so thankful to Dr. R. Watson for his precise English revision.

\section{Authors' contributions \\ Research idea and study design: AKY, FK, MM; data acquisition: FK; data analysis/interpretation: AKY, MM; statistical analysis: AKY; supervision or mentorship: MM, SEJ. Each author contributed important intellectual content during manuscript drafting or revision, accepts personal accountability for the author's own contributions, and agrees to ensure that questions pertaining to the accuracy or integrity of any portion of the work are appropriately investigated and resolved. All authors read and approved the final manuscript.}

\section{Funding}

This study was granted by the vice chancellery of Shiraz University of Medical Sciences, Shiraz, Iran. The funder had no involvement in the design of the study and collection, analysis, and interpretation of data and in writing the manuscript.

\section{Availability of data and materials}

All data generated or analysed during this study are included in this published article [and its supplementary information files (additional file 1)].

\section{Ethics approval and consent to participate}

The study was approved by the Ethics Committee of the vice chancellery of Shiraz University of Medical Sciences in concordance with the standards of Helsinki declaration (Ethics number: IR.SUMS.REC.1396.2.9). All the participants signed a written informed consent form before the study commences.

\section{Consent for publication}

Not Applicable.

\section{Competing interests}

The authors declare there are no competing interests.

\section{Author details}

${ }^{1}$ Physical therapy Department, School of Rehabilitation Sciences, Shiraz University of Medical Sciences, Shiraz, Iran. ${ }^{2}$ Student Research Committee, School of Nursing and Midwifery, Shiraz University of Medical Sciences, Shiraz, Iran. ${ }^{3}$ Department of Nursing, School of Nursing and Midwifery, Shiraz University of Medical Sciences, Zand St., Namazee Sq, Shiraz 7193613119, Iran. ${ }^{4}$ Nephro-urology research center, Shiraz University of Medical Sciences, Shiraz, Iran.

Received: 13 June 2020 Accepted: 23 December 2020

Published online: 07 January 2021

\section{References}

1. Rehman IU, Khan TM. Validity and reliability of the Urdu version of the 5D itching scale to assess pruritus among patients with chronic kidney disease in Pakistan. BMC Nephrol. 2017;18(1):302.

2. Schwartz IF. Uremic pruritus. Nephrol Dial Transplant. 1999;14:834-9.

3. Simonsen $E$, Komenda P, Lerner B, et al. Treatment of uremic pruritus: a systematic review. Am J Kidney Dis. 2017;70(5):638-55.

4. Khorsand A, Salari R, Noras MR, et al. The effect of massage and topical violet oil on the severity of pruritus and dry skin in hemodialysis patients: a randomized controlled trial. Complementary therapies in medicine. 2019;45: 248-53.

5. Lopes GB, Nogueira FCP, de Souza MR, et al. Assessment of the psychological burden associated with pruritus in hemodialysis patients using the kidney disease quality of life short form. Qual Life Res. 2012;21(4): 603-12.

6. Darsow U, Mautner $\mathrm{V}$, Bromm B, et al. The Eppendorf pruritus questionnaire. Der Hautarzt. Zeitschrift fur Dermatologie, Venerologie, und verwandte Gebiete. 1997;48(10):730-3.

7. Chren M-M. The Skindex instruments to measure the effects of skin disease on quality of life. Dermatol Clin. 2012;30(2):231-6.

8. Wells KB, Stewart A, Hays RD, et al. The functioning and well-being of depressed patients: results from the medical outcomes study. Jama. 1989; 262(7):914-9.

9. Elman S, Hynan L, Gabriel V, et al. The 5-D itch scale: a new measure of pruritus. Br J Dermatol. 2010;162(3):587-93.

10. Ersoy NA, Akyar I. Multidimensional pruritus assessment in hemodialysis patients. BMC Nephrol. 2019;20(1):42.

11. Hair J, Black W, Babin B, et al. Multivariate data analysis. Upper Saddle River, NJ [etc.]. Pearson Prentice Hall, New York, NY: Macmillan. 2009;24:899.

12. Beaton DE, Bombardier C, Guillemin F, et al. Guidelines for the process of cross-cultural adaptation of self-report measures. Spine. 2000;25(24):3186-91.

13. Yoosefinejad A, Hadadi M, Eslamloo P. EVALUATING THE RESPONSIVENESS OF THE FULLERTON ADVANCED BALANCE SCALE IN PATIENTS WITH LYMP HEDEMA SECONDARY TO BREAST CANCER SURGERY. Lymphology. 2019; 52(2):61-70

14. Lai J-W, Chen H-C, Chou C-Y, et al. Transformation of 5-D itch scale and numerical rating scale in chronic hemodialysis patients. BMC Nephrol. 2017; 18(1):56.

15. Desai NS, Poindexter GB, Monthrope YM, et al. A pilot quality-of-life instrument for pruritus. J Am Acad Dermatol. 2008;59(2):234-44.

16. Tari N, Mahmoodi H, Hosseini SH, et al. Structural reliability and validity of Persian translation of. Journal of Dermatology and Cosmetic. 2012;3(2):92-9. 
17. Ghasemi F, Yoosefinejad AK, Pirouzi S, et al. Evaluating the cross-cultural adaptation, reliability, and validity of the Persian versions of the Copenhagen neck function disability scale and neck Bournemouth questionnaire. Spine. 2019;44(2):E126-32.

18. Farazdaghi MR, Mansoori A, Vosoughi O, et al. Evaluation of the reliability and validity of the Persian version of patient-rated elbow evaluation questionnaire. Rheumatol Int. 2017;37(5):743-50.

19. Kanyongo GYJJoMASM. Determining the correct number of components to extract from a principal components analysis: A Monte Carlo study of the accuracy of the scree plot. 2005;4(1):13.

20. Wulandani MP, Dachlan AS, Yusharyahya SN. Validity and reliability of 5-D itch scale in Indonesian language on adult and geriatric patient at Dr. Cipto Mangunkusumo Hospital Advanced Science Letters. 2018;24(9):6994-8.

21. Rehman IU, WU DB-C, Lai P, et al. Translation of the $5 D$ itching scale from english to Malay, and its validation among patients with chronic kidney disease in Malaysia. Frontiers in medicine. 2017:4:189.

22. Khan TM, Al-Haider I, Syed Sulaiman SA, et al. Linguistic validation of the $5 D$ itching scale to Arabic in patients with end-stage kidney disease. Journal of renal care. 2013;39(4):222-7.

23. Takahashi N, Yoshizawa T, Okubo A, et al. Usefulness of the Japanese version of the $5-D$ itch scale for rating pruritus experienced by patients undergoing hemodialysis. Renal Replacement Therapy. 2018;4(1):26.

\section{Publisher's Note}

Springer Nature remains neutral with regard to jurisdictional claims in published maps and institutional affiliations.

Ready to submit your research? Choose BMC and benefit from:

- fast, convenient online submission

- thorough peer review by experienced researchers in your field

- rapid publication on acceptance

- support for research data, including large and complex data types

- gold Open Access which fosters wider collaboration and increased citations

- maximum visibility for your research: over $100 \mathrm{M}$ website views per year

At BMC, research is always in progress.

Learn more biomedcentral.com/submissions 